\title{
Transdisciplinariedad en el abordaje de la amiloidosis
}

\author{
Transdisciplinarity in the approach to amyloidosis
}

Transdisciplinaridade na abordagem da amiloidose

\section{Amalia Ernestina Alfonsín ${ }^{1}$, Elsa Mercedes Nucifora ${ }^{2}$, María Adela Aguirre ${ }^{3}$. María Lourdes Posadas MartíneZ Melina Paula Valeo Chulvi ${ }^{5}$, Dana Kohan ${ }^{6}$, Sofía Stephanie Escada ${ }^{7}$}

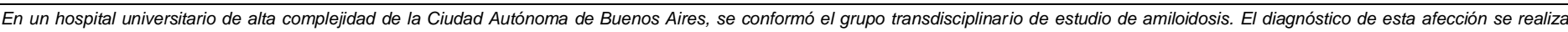

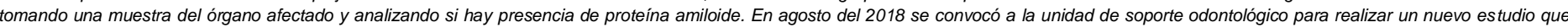

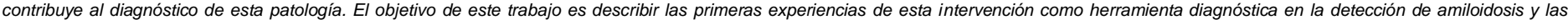
complicaciones que puedan ocurrir antes o después del procedimiento.

\section{Conceptos clave:}

El diagnóstico de amiloidosis se basa en la biopsia del órgano afectado: corazón, riñón, hígado, bazo, sistema nervioso 0 tracto digestivo. Este procedimiento es invasivo, puede provocar un sangrado y complicar el estado general del paciente ya comprometido por la enfermedad. Por ello, las biopsias de glándulas salivales menores han sido reportadas como alternativa de baja complejidad y bien tolerada por los pacientes. Este trabajo describe nuestras experiencias y resultados con el objetivo de contribuir al incremento del conocimiento en esta área

1- Doctora en Odontología (UBA), Coordinadora de la Unidad de Soporte Odontológico, Odontóloga auditora Plan de Salud, Investigadora Adscripta, Profesora Instituto Universitario, Integrante Grupo de Amiloidosis. Lugar de trabajo: Hospital Italiano de Buenos Aires. Orcid: https://orcid.org/0000-0002 5399-0197 E-mail de contacto: amalia.alfonsin@hospitalitaliano.org.ar

2- Médica (UBA) Especialista en hematología, Médica asociada del Servicio de Clínica Médica, Médica del Staff de Hematología Directora de la carrera de especialista en hematola Inegrante Grupo de Amiloidosis. Hospital hematologia, integrante Aires. Orcid: https://orcid.org/0000-0003$\underline{2177-7727}$

3- Médica (USAL) Profesora Instituto Universitario. Coordinadora Centro Simulación de habilidades quirúrgicas del Instituto Universitario, Médica de planta del servicio de Clínica Médica, Integrante Grupo de Amiloidosis. Lugar de trabajo: Imtib y Servicio de Clínica médica del Hospital Italiano de Bs As. Orcid: https://orcid.org/0000-0001-9690-2284

4- Médica (Universidad Austral). Estadística (UBA), Magister en Metodología de la Investigación (Universidad de Lanus) Investigador asistente del Conicet. Investigador staff del área de investigación de Medicina Interna HIBA, Investigador Formado HIBA, Integrante Grupo de Amiloidosis Lugar de trabajo: Hospital Italiano de Buenos Aires, Conicet. Orcid: https://orcid.org/0000-0003-1403-7069

5- Médica (UBA) Residencia Anatomía Patológica. Actual Jefa de residentes del servicio de Anatomía patológica del HIBA. Integrante Grupo de Amiloidosis. Lugar de trabajo: Hospital Italiano de Buenos Aires.

6- Médica (UBA) Especialista en patología y citología. Médica asociada del Servicio de Anatomía Patológica. Especialista y magíster en Economía y Gestión de Salud., Integrante Grupo de Amiloidosis. Lugar de trabajo: Hospital Italiano de Buenos Aires.

7- Odontóloga (UBA) Integrante equipo Unidad Soporte Odontológico, Docente invitada Instituto Universitario, Becaria de Perfeccionamiento en auditoría y gestión de los servicios odontológicos, Integrante Grupo de Amiloidosis. Lugar de odontológicos, Integrante Grupo de Amiloidosis. Lugar de
trabajo: Hospital Italiano de Buenos Aires. Orcid: https://orcid.org/0000-0002-2278-2950

Recibido: 2021-03-04 Aceptado: 2021-05-21

DOI: http://dx.doi.org/10.31053/1853.0605.v77.n4.32341

\section{Resumen:}

La creciente complejidad en el abordaje de las enfermedades desafía a desarrollar un nuevo paradigma de atención que atraviese los límites disciplinarios, donde profesionales de diferentes especialidades abordan un problema aplicando su expertise, respetando el conocimiento del otro y contribuyendo a la mirada global de la problemática.

Las amiloidosis son enfermedades raras. El diagnóstico se basa en la biopsia de aquellos órganos en los que se sospecha de infiltración amiloidea. Existe un aumento del riesgo de sangrado asociado con estos procedimientos. Por ello, la disponibilidad de obtener muestras de determinados sitios con accesos menos invasivos, como las glándulas salivales menores (GSM), representan una alternativa. Objetivos: Describir las experiencias de biopsias GSM como herramienta diagnóstica en la detección de amiloidosis y sus complicaciones intra y postoperatorias. Estimar la performance diagnóstica. El diseño es de cohorte transversal. Se incluyeron consecutivamente todos los pacientes con sospecha de diagnóstico de amiloidosis, entre agosto 2018-septiembre 2020. Resultados: Se efectuaron 23 biopsias con procedimientos mínimamente invasivos. El 60,86 \% fueron de sexo masculino y la media de edad 66 años. La prevalencia de amiloidosis fue del $33 \%$. La sensibilidad fue del $55 \%$ y la especificidad del $100 \%$. Un paciente presentó mayor sangrado que lo habitual durante el procedimiento y ninguno presentó complicaciones postoperatorias. Conclusiones: Se describieron las experiencias de biopsia de GSM como herramienta diagnóstica de amiloidosis, reportando una leve complicación intraoperatoria y nula posoperatoria. La prueba alcanzó una sensibilidad del $55 \%$ y una especificidad del $100 \%$.

Palabras claves: amiloidosis; glándulas salivales; biopsia

\section{Abstract:}

The increasing complexity in the approach to diseases challenges the development of a new paradigm of care that crosses disciplinary limits, where professionals from different disciplines approach a problem applying their expertise, respecting the knowledge of the other and contributing to the global view of the problem. Amyloidoses are rare diseases. The diagnosis is based on the biopsy of those organs in which amyloid infiltration is suspected. There is an increased risk of bleeding associated with these procedures. Therefore, the availability of obtaining samples from certain sites with less invasive accesses, such as the minor salivary glands (GSM), represent an alternative. Objectives: Describe the experiences of GSM biopsies as a diagnostic tool in the detection of amyloidosis and its intra and postoperative complications. - Estimate the diagnostic performance. The design is a cross-sectional cohort. All patients with a suspected diagnosis of amyloidosis were consecutively included, between August 2018-September 2020. Results: 23 biopsies were performed with minimally invasive procedures. $60.86 \%$ were male and the average age was 66 years. The prevalence of amyloidosis was 33\%. The sensitivity was $55 \%$ and the specificity $100 \%$. One patient had more bleeding than usual during the procedure and none had postoperative complications. Conclusions: GSM biopsy experiences were described as a diagnostic tool for amyloidosis, reporting a slight intraoperative complication and no postoperative complication. The test achieved a sensitivity of $55 \%$ and a specificity of $100 \%$

Keywords: amyloidosis; salivary glands; biopsy

\section{Resumo}

A crescente complexidade na abordagem das doenças desafia o desenvolvimento de um novo paradigma de cuidado que extrapola os limites disciplinares, onde profissionais de diferentes disciplinas abordam um problema aplicando suas competências, respeitando o conhecimento do outro e contribuindo para a visão global do problema. As amiloidoses são doenças raras. $\mathrm{O}$ diagnóstico é baseado na biópsia dos órgãos em que há suspeita de infiltração amilóide. Há um risco aumentado de sangramento associado a esses procedimentos. Portanto, a disponibilidade de obtenção de amostras em determinados locais com acessos menos invasivos, como as glândulas salivares menores (GSM), representa uma alternativa. Metas: Descrever as experiências das biópsias GSM como ferramenta diagnóstica na detecção da amiloidose e suas complicações intra e pós-operatórias. - Estimar o desempenho diagnóstico. O desenho é uma coorte transversal. Todos os pacientes com suspeita de diagnóstico de amiloidose foram incluídos consecutivamente, entre agosto de 2018 e setembro de 2020 . Resultados: 23 biópsias foram realizadas com procedimentos minimamente invasivos. $60,86 \%$ eram do sexo masculino e a idade média era de 66 anos. A prevalência de amiloidose foi de 33\%. A sensibilidade foi de 55\% e a especificidade de $100 \%$. Um paciente teve mais sangramento que o normal durante o procedimento e nenhum apresentou complicações pós-operatórias. Conclusões: Foram descritas experiências de biópsia GSM como ferramenta diagnóstica para amiloidose, relatando uma leve complicação intra-operatória e nenhuma complicação pós-operatória. O teste atingiu uma sensibilidade de $55 \%$ e uma especificidade de $100 \%$ 


\section{INTRODUCCIÓN}

La creciente complejidad en el abordaje de las enfermedades desafía a desarrollar un nuevo paradigma de atención que atraviese los límites disciplinarios, donde profesionales de diferentes especialidades abordan un problema aplicando su expertise, respetando el conocimiento del otro y contribuyendo a la mirada global de la problemática ${ }^{(1)}$. Las amiloidosis sistémicas son enfermedades raras. La incidencia mínima estimada de amiloidosis sistémica en la población inglesa en 2008 fue de 4 por 1000000 personas-año y alcanzó su punto máximo entre los 60 y 79 años (2) Se caracteriza por el depósito extracelular de fibrillas amiloides insolubles ${ }^{(3)}$. La nomenclatura de la enfermedad está basada en los precursores de estas fibrillas y se han identificado al menos 30 proteínas diferentes (4). Dependiendo la naturaleza de la proteína y el sitio de deposición, se clasifican en amiloidosis primaria $(A L)$, inmunoglobulina cadenas livianas; amiloidosis secundaria (AA) proteína A amiloide; amiloidosis transtiretina (TTR).

El diagnóstico se basa en la biopsia de aquellos órganos en los que se sospecha de infiltración amiloidea ${ }^{(5)}$. La tinción histoquímica clásica para el amiloide es rojo Congo, introducida por Bennhold en $1922^{(6) ;}$ la birrefringencia usando polarización típica 'verde manzana' fue descrita

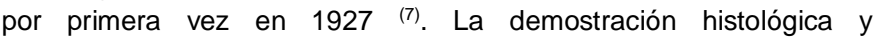
caracterización precisa del depósito de amiloide es fundamental en su diagnóstico. ${ }^{(8,9)}$

Existe un aumento del riesgo de sangrado reportado asociado con estos procedimientos ${ }^{(10)}$. Por ello, la disponibilidad de obtener muestras de sitios cuyo acceso es menos invasivo, como grasa abdominal y glándulas salivales menores (GSM), representan una alternativa a la biopsia de órganos. Las GSM, se encuentran distribuidas en la mucosa bucal. Se designan de acuerdo a su ubicación como: labiales, genianas, palatinas y linguales. Son pequeñas y se estima que existen entre 450 a 800 , localizadas muy próximas a la superficie interna de la boca. Están constituidas por numerosos acúmulos acinares, compuesto de pequeños y cortos cordones excretores que se abren en la cara interna de los labios. La presencia de estas glándulas confiere un aspecto granular a la superficie de la mucosa labial. Esta localización permite gran accesibilidad para la toma de muestras, contribuyendo al diagnóstico de la amiloidosis, y de otras enfermedades como el Síndrome de Sjogren o la sarcoidosis ${ }^{(11) .}$ Estudios previos reportaron la biopsia de GSM como método diagnóstico de amiloidosis poco invasivo y de baja complejidad ${ }^{(12,13,14)}$

En un hospital de alta complejidad de CABA se conformó el grupo de estudio de amiloidosis (GEA) con criterio de transdisciplinariedad. Incluye bioquímicos, médicos clínicos, hematólogos, nefrólogos, cardiólogos, oftalmólogos, investigadores, hepatólogos, anatomopatólogos, neurólogos y dermatólogos. En agosto 2018, convocó a la Unidad de Odontología para incorporar las biopsias de GSM como herramienta diagnóstica.

Los objetivos de este trabajo fueron:

Describir las primeras experiencias transdisciplinarias de biopsias GSM como herramienta diagnóstica de amiloidosis

Describir las complicaciones intra y postoperatorias.

Estimar la performance diagnóstica

\section{MÉTODOS}

El diseño es de cohorte transversal. Se incluyeron consecutivamente los pacientes con sospecha de diagnóstico de amiloidosis con biopsia de glándula salival del Registro Institucional de Amiloidosis (RIA) del hospital. Período agosto 2018- septiembre 2020. Este registro presenta reclutamiento activo desde el 2010, prospectivo, de todos los casos incidentes de amiloidosis. Funciona transdisciplinariamente, con la participación del Área de Investigación en Medicina Interna, Anatomía patológica, Laboratorio, Cardiología, Clínica médica, Hematología, Hepatología, Odontología, Nefrología y Neurología. Está registrado en Clinical Trials (NCT01347047) y aprobado por el comité de ética de

protocolos de investigación del hospital con el $\mathrm{N}^{\circ}$ de protocolo 1675. Los pacientes realizan el proceso de consentimiento informado antes de ser incluidos en el registro. La Unidad de Soporte Odontológico (USO) se conformó en febrero 2015 y tiene el objetivo de eliminar los focos sépticos de la cavidad bucal de pacientes internados y ambulatorios con enfermedades asociadas, que estén bajo atención médica en el mismo hospital. Trabaja transdisciplinariamente con los equipos médicos tratantes e incorpora sus evoluciones a la historia clínica electrónica, permitiendo la integración de sus procedimientos y favoreciendo los cuidados centrados en el paciente.

Los pacientes con sospecha de amiloidosis son derivados a la USO donde se procede a la toma de biopsia que consta de los siguientes pasos:

- $\quad$ Se constata que se encuentren en condiciones sistémicas de afrontar el procedimiento previa evaluación de los parámetros de coagulación.

- $\quad$ Explicación del procedimiento y firma del consentimiento informado.

- Marcado quirúrgico de la zona

- Desinfección de la mucosa del labio inferior con clorhexidina $0,12 \%$.

- Infiltración local con anestésico para uso odontológico ( carticaína clorhidrato 4\% -adrenalina 1:100.000.)

- Incisión lineal con bisturí frío. Las GSM se localizan mediante inspección visual. Se resecan con pinza de disección tipo Adson atraumática. (foto 1)

- $\quad$ Sutura con puntos simples con Vicryl 4-0 poliglactina 910 reabsorbible.

- Indicaciones y cuidados posoperatorios

- Las muestras son fijadas en formol $10 \%$ y se remiten al servicio de anatomía patológica para ser procesadas (foto 2)

- A los 7 días: control postoperatorio del paciente y retiro de puntos de sutura.

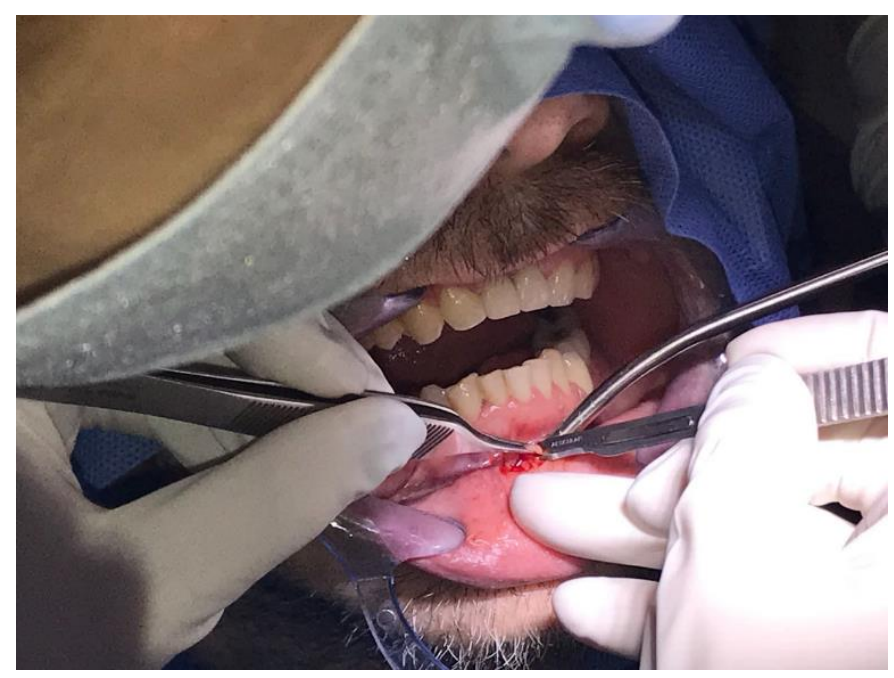

Foto 1: Toma de biopsia 


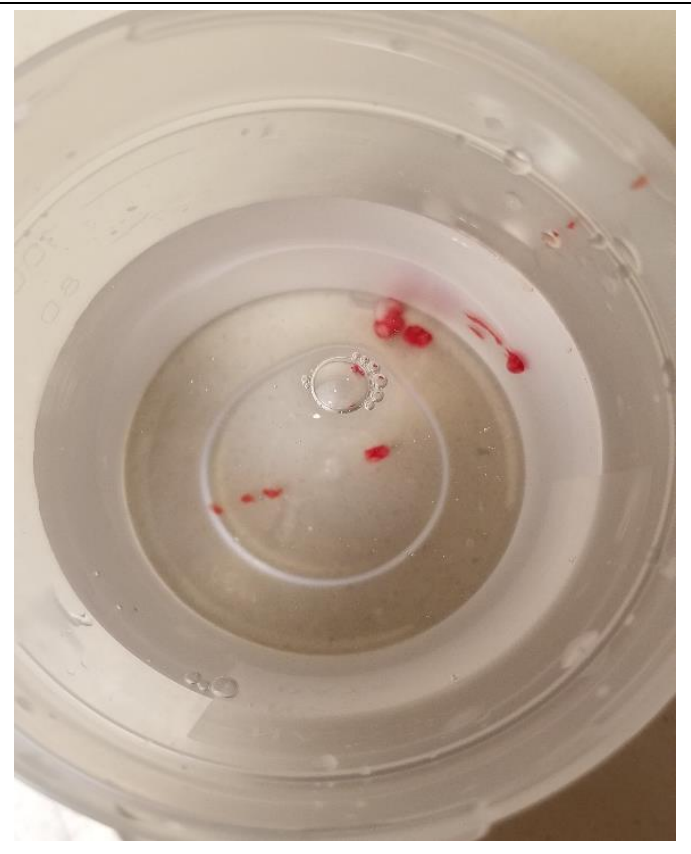

Foto 2. Muestra glándulas salivales

\section{Procedimiento en anatomía patológica}

Evaluación microscópica. El examen del Rojo Congo es poco sensible y los depósitos tempranos de amiloide pueden ser escasos o pueden estar distribuidos en forma no uniforme. La muestra no debe estar teñida con exceso de colorante que lleve a falsos positivos, el grosor debe ser adecuado y se debe trabajar con una muestra testigo. Es obligatorio ver el material Rojo Congo positivo bajo el efecto de la luz polarizada para confirmar el diagnóstico. La Tioflavina T puede ayudar a confirmar o descartar un diagnóstico histológico dudoso ${ }^{(15)}$. Los pacientes fueron clasificados en positivo o negativo para amiloidosis si presentaba tinción roja congo +. Se consideró el test de referencia (gold standard) a la biopsia de otro órgano positivo para amiloidosis o la combinación de estudios diagnósticos confirmatorios de amiloidosis (cadenas livianas+ife sérica y urinaria+imagen cardiaca sugestiva de amiloidosis) (CITA DORbala). Se recolectó información clínico demográfica: edad, sexo y referidas a la cavidad bucal:

Previo a la biopsia:

- Hiposalivación o xerostomía

- Macroglosia

- Dificultad para tragar y/o masticar

- Coloración amarillenta mucosa

- Adenopatía submandibular

Complicación intra biopsia:

- $\quad$ Sangrado mayor a lo habitual durante el procedimiento de toma de biopsia

Complicación postoperatoria:

- Hematoma

Análisis estadístico: Las variables cuantitativas fueron descritas con su media y desvío estándar o mediana e intervalo intercuartil según distribución. Las categóricas se reportaron como frecuencias y porcentajes. La prevalencia de amiloidosis se estimó como porcentaje y se calcularon los intervalos de confianza del 95\% (IC95\%).

Este trabajo fue eximido de la necesidad de revisión por el Comité de Ética del hospital, sin embargo, se han respetado los principios de la Declaración de Helsinski.

\section{RESULTADOS}

Se incluyeron 23 biopsias con procedimientos mínimamente invasivos. El $60,86 \%$ fueron de sexo masculino, y la media de edad 66 años (rango 40-87). La prevalencia de amiloidosis fue del $33 \%$ ( $n=9$, IC 17$90 \%$ ). La exactitud global de la prueba $85,19 \%$ (IC 65-96\%). La sensibilidad alcanzó el $55 \%(21,20 \%-86,30 \%)$ y la especificidad el 100 $\%(81,47 \%-100 \%)$, el valor predictivo positivo $100 \%$, y el valor predictivo negativo $82 \%(68,43 \%$ - 90.33\%). El 4,34 \% de los pacientes presentaron hiposalivación, dificultad para masticar o tragar y adenopatía submandibular. El 8,69\% presentaron macroglosia y color amarillento de mucosas. El porcentaje de complicaciones intraoperatorias fue de $4 \%$ ( $n=1$, IC $0-12 \%$ ) y se reportó como sangrado levemente superior a lo habitual. El porcentaje de complicaciones postoperatorias (hematomas) fue del $0 \%$. Se perdió el seguimiento de $4 / 23$ que eran pacientes del interior del país. Se les indicó informar si presentaron complicaciones, sin embargo, no se recibió ningún reporte. Discusión / conclusión:

Este trabajo describió la experiencia transdisciplinaria de biopsias (GSM) como herramienta diagnóstica en la detección de amiloidosis, las complicaciones intra y postoperatorias y se estimó su performance diagnóstica.

Si bien la sensibilidad de la biopsia de GSM en nuestro centro es levemente inferior a la reportada en otros trabajos ${ }^{(15,16,17)}$, esto podría deberse al escaso número de pacientes incluidos, que representa la limitación principal de este trabajo. Para obtener resultados más determinantes, el equipo actualmente continúa incorporando nuevos casos. En esta enfermedad es importante valorar al paciente globalmente y para ello, es importante contar con una sumatoria de tests diagnósticos que aumenten la sensibilidad. La transdisciplinariedad es crucial en el diagnóstico de pacientes con enfermedades poco frecuentes con compromiso sistémico como la amiloidosis. La inclusión de las biopsias de GSM dentro de la evaluación inicial del paciente con sospecha de amiloidosis representa un avance en el acortamiento del tiempo del proceso diagnóstico. Además, resultó ser una técnica mínimamente invasiva y bien aceptada por los pacientes, especialmente en aquellos severamente comprometidos por la enfermedad. Por estos motivos, consideramos que la amiloidosis amerita este tipo de trabajo donde múltiples especialidades comparten su expertise y permite discusiones individuales para establecer con certeza el diagnóstico.

Se describieron las experiencias de biopsia de GSM como herramienta diagnóstica de amiloidosis, reportando baja complicación intraoperatoria y nula posoperatoria. La prueba alcanzó una sensibilidad del $55 \%$ y una especificidad del $100 \%$

Limitaciones de responsabilidad:

La responsabilidad de este trabajo es exclusivamente de los autores.

Conflicto de interés:

Ninguno

Fuentes de apoyo:

La presente investigación no contó con fuentes de financiación.

Originalidad del trabajo:

Este artículo es original y no ha sido enviado para su publicación a otro medio de difusión científica en forma completa ni parcialmente.

Cesión de derechos:

Los participantes de este trabajo ceden el derecho de autor a la Universidad Nacional de Córdoba para publicar en la RFCM y realizar las traducciones necesarias al idioma inglés.

Contribución de los autores:

Todos los autores hemos participado en la concepción del diseño, recolección de la información y elaboración del manuscrito, haciéndose públicamente responsables de su contenido y aprobando su versión final. 


\section{BIBLIOGRAFÍA}

1.Aguirre MA, Boietti BR, Nucifora E, Sorroche PB, González Bernaldo de Quirós F, Giunta $D H$, Posadas-Martínez ML. Incidence rate of amyloidosis in patients from a medical care program in Buenos Aires, Argentina: a prospective cohort. Amyloid. 2016 Sep;23(3):184-187. doi: 10.1080/13506129.2016.1207626.

2.Pinney JH, Smith CJ, Taube JB, Lachmann HJ, Venner CP, Gibbs $S D$, Dungu J, Banypersad SM, Wechalekar AD, Whelan CJ, Hawkins $P N$, Gillmore JD. Systemic amyloidosis in England: an epidemiological study. Br J Haematol. 2013 May;161(4):525-32. doi: 10.1111/bjh. 12286. 3. Chiti F, Dobson CM. Protein Misfolding, Amyloid Formation, and Human Disease: A Summary of Progress Over the Last Decade. Annu Rev Biochem. 2017 Jun 20;86:27-68. doi: 10.1146/annurev-biochem061516-045115.

4. Sipe JD, Benson MD, Buxbaum JN, Ikeda S, Merlini G, Saraiva MJ, Westermark P. Nomenclature 2014: Amyloid fibril proteins and clinical classification of the amyloidosis. Amyloid. 2014 Dec;21(4):221-4. doi: 10.3109/13506129.2014.964858.

5. Lado Lado FL, Ferreiro Regueiro M J, Cabana González B, Díez Díez $V$, Maceda Vilariño S, Antúnez López J R. Amiloidosis. Medicina Integral 2000;137-141.

6. Bennhold H: Specific staining of amyloid by Congo red. Munch Med Wochenschr 1922; 69: 1537-1538.

7. Divry P, Florkin M: Sur les propriétés optiques de l'amyloide. Paris, CR Société de Biologie, 1927, pp 1808-1810.

8. Lachmann HJ, Booth DR, Booth SE, Bybee A, Gilbertson JA, Gillmore $J D$, Pepys MB, Hawkins PN. Misdiagnosis of hereditary amyloidosis as AL (primary) amyloidosis. N Engl J Med. 2002 Jun 6;346(23):1786-91. doi: 10.1056/NEJMoa013354.

9. Gertz MA, Comenzo R, Falk RH, Fermand JP, Hazenberg BP, Hawkins PN, Merlini G, Moreau P, Ronco P, Sanchorawala V, Sezer O, Solomon A, Grateau G. Definition of organ involvement and treatment response in immunoglobulin light chain amyloidosis ( $A L)$ : a consensus opinion from the 10th International Symposium on Amyloid and Amyloidosis, Tours, France, 18-22 April 2004. Am J Hematol. 2005 Aug;79(4):319-28. doi: 10.1002/ajh.20381.

10. Soares SM, Fervenza FC, Lager DJ, Gertz MA, Cosio FG, Leung N. Bleeding complications after transcutaneous kidney biopsy in patients with systemic amyloidosis: single-center experience in 101 patients. Am J Kidney Dis. 2008 Dec;52(6):1079-83. doi: 10.1053/j.ajkd.2008.05.022. 11. Lida Santiago M, Seisdedos MR, García Salinas RN, Secco A, Marino Claverie L, Techera L, Takashima L, Aicardi P, Sandi Rosales MA, Knobel E, Magri SJ, Catalán Pellet AC. Frequency of complications and usefulness of the minor salivary gland biopsy. Reumatol Clin. 2012 Sep-Oct;8(5):255-8. doi: 10.1016/j.reuma.2012.03.006.

12 Lecadet A, Bachmeyer C, Buob D, Cez A, Georgin-Lavialle S. Minor salivary gland biopsy is more effective than normal appearing skin biopsy for amyloid detection in systemic amyloidosis: A prospective monocentric study. Eur J Intern Med. 2018 Nov;57:e20-e21. doi: 10.1016/j.ejim.2018.07.026.

13. de Paula Eduardo F, de Mello Bezinelli L, de Carvalho DL, DellaGuardia B, de Almeida MD, Marins LV, Corrêa L. Minor salivary gland biopsy for the diagnosis of familial amyloid polyneuropathy. Neurol Sci. 2017 Feb;38(2):311-318. doi: 10.1007/s10072-016-2760-1.

14. Suzuki T, Kusumoto S, Yamashita T, Masuda A, Kinoshita S, Yoshida T, Takami-Mori F, Takino H, Ito A, Ri M, Ishida T, Komatsu H, Ueda $M$, Ando $Y$, Inagaki $H$, lida $S$. Labial salivary gland biopsy for diagnosing immunoglobulin light chain amyloidosis: a retrospective analysis. Ann Hematol. 2016 Jan;95(2):279-85. doi: 10.1007/s00277015-2549-y.

15. Linke RP. Protein Misfolding, Aggregation, and Conformational Diseases. Boston: Springer; 2006. Chapter 1, Congo Red Staining of Amyloid: Improvements and Practical Guide for a More Precise Diagnosis of Amyloid and the Different Amyloidoses; $p$ 239-276. 